\title{
Representation by Degenerate Genocchi Polynomials
}

\author{
Taekyun Kim $\mathbb{D}^{1},{ }^{1}$ Dae San Kim $\mathbb{D}^{2},{ }^{2}$ Jongkyum Kwon $\mathbb{D}^{3},{ }^{3}$ and Seong-Ho Park $\mathbb{D}^{1}$ \\ ${ }^{1}$ Department of Mathematics, Kwangwoon University, Seoul 139-701, Republic of Korea \\ ${ }^{2}$ Department of Mathematics, Sogang University, Seoul 121-742, Republic of Korea \\ ${ }^{3}$ Department of Mathematics Education, Gyeongsang National University, Jinju 52828, Republic of Korea
}

Correspondence should be addressed to Jongkyum Kwon; mathkjk26@gnu.ac.kr

Received 21 July 2021; Revised 13 November 2021; Accepted 7 January 2022; Published 21 January 2022

Academic Editor: Yuan Yi

Copyright ( $) 2022$ Taekyun Kim et al. This is an open access article distributed under the Creative Commons Attribution License, which permits unrestricted use, distribution, and reproduction in any medium, provided the original work is properly cited.

The aim of this study is to represent any polynomial in terms of the degenerate Genocchi polynomials and more generally of the higher-order degenerate Genocchi polynomials. We derive explicit formulas with the help of umbral calculus and illustrate our results with some examples.

\section{Introduction and Preliminaries}

In this study, we derive formulas expressing any polynomial in terms of the degenerate Genocchi polynomials ((14)) with the help of umbral calculus (Theorem 1) and illustrate our results with some examples (Section 5). This can be generalized to the higher-order degenerate Genocchi polynomials $((15))$. Indeed, we deduce formulas for representing any polynomial in terms of the higher-order degenerate Genocchi polynomials again by using umbral calculus (Theorem 2). The contribution of this study is the derivation of such formulas which have potential applications to finding some interesting polynomial identities, as illustrated in Section 5.

Many interesting identities have been derived by using similar formulas for representations by Bernoulli, Euler, and Frobenius-Euler polynomials (see [1-9]). The list in the references is far from being exhaustive. However, the interested reader can easily find more related studies in the literature. Also, we should mention here that there are other ways of obtaining the same result as the one in (2). One of them is to use Fourier series expansion of the function obtained by extending by periodicity of period 1 of the polynomial function restricted to the interval $[0,1)$ [10].
Let $p(x) \in \mathbb{C}[x]$, with $\operatorname{deg} p(x)=n$. Write $p(x)=\sum_{k=0}^{n}$ $a_{k} B_{k}(x)$, where $B_{n}(x)$ are the Bernoulli polynomials defined by $\left(t / e^{t}-1\right) e^{x t}=\sum_{n=0}^{\infty} B_{n}(x)\left(t^{n} / n !\right)$. Then, it is known that $a_{0}=\int_{0}^{1} p(t) \mathrm{d} t$ $a_{k}=\frac{1}{k !}\left(p^{(k-1)}(1)-p^{(k-1)}(0)\right), \quad$ for $k=1,2, \ldots, n$.

Applying the formulas in (1) to the polynomial $p(x)=\sum_{k=1}^{n-1} 1 / k(n-k) B_{k}(x) B_{n-k}(x)$, we can get an identity which yields, after slight modification, the following identity:

$$
\begin{aligned}
& \sum_{k=1}^{n-1} \frac{1}{2 k(2 n-2 k)} B_{2 k}(x) B_{2 n-2 k}(x)+\frac{2}{2 n-1} B_{1}(x) B_{2 n-1}(x) \\
& =\frac{1}{n} \sum_{k=1}^{n} \frac{1}{2 k}\left(\begin{array}{c}
2 n \\
2 k
\end{array}\right) B_{2 k} B_{2 n-2 k}(x)+\frac{1}{n} H_{2 n-1} B_{2 n}(x) \\
& \quad+\frac{2}{2 n-1} B_{1}(x) B_{2 n-1}
\end{aligned}
$$

where $n \geq 2$, and $H_{n}=1+(1 / 2)+\cdots+(1 / n)$.

Letting $x=0$ in (2), we obtain a slight variant of the wellknown Miki's identity: 


$$
\begin{aligned}
& \sum_{k=1}^{n-1} \frac{1}{2 k(2 n-2 k)} B_{2 k} B_{2 n-2 k}=\frac{1}{n} \sum_{k=1}^{n} \frac{1}{2 k}\left(\begin{array}{c}
2 n \\
2 k
\end{array}\right) B_{2 k} B_{2 n-2 k} \\
& +\frac{1}{n} H_{2 n-1} B_{2 n} \text {. }
\end{aligned}
$$

Letting $x=1 / 2$ in (2), we get the Faber-Pandharipande-Zagier (FPZ) identity:

$$
\begin{aligned}
\sum_{k=1}^{n-1} \frac{1}{2 k(2 n-2 k)} \bar{B}_{2 k} \bar{B}_{2 n-2 k}= & \frac{1}{n} \sum_{k=1}^{n} \frac{1}{2 k}\left(\begin{array}{c}
2 n \\
2 k
\end{array}\right) B_{2 k} \bar{B}_{2 n-2 k} \\
& +\frac{1}{n} H_{2 n-1} \bar{B}_{2 n},
\end{aligned}
$$

where $\bar{B}_{n}=\left(2^{1-n}-1\right) B_{n}=B_{n}(1 / 2)$, for all $n=0,1,2, \ldots$

Here, it should be stressed that the other proofs of Miki's ([11-13]) and FPZ identities ([14,15]) are quite involved, while our proofs of Miki's and Faber-Pandharipande-Zagier identities follow from the simple formulas in (1) involving only derivatives and integrals of the given polynomials.

The outline of this study is as follows. In Section 1, we recall some necessary facts that are needed throughout this study. In Section 2, we go over umbral calculus briefly. In Section 3, we derive formulas expressing any polynomial in terms of the degenerate Genocchi polynomials. In Section 4, we derive formulas representing any polynomial in terms of the higher-order degenerate Genocchi polynomials. In Section 5, we illustrate our results with some examples. Finally, we conclude our study in Section 6.

The Euler polynomials $E_{n}(x)$ are defined by

$$
\frac{2}{e^{t}+1} e^{x t}=\sum_{n=0}^{\infty} E_{n}(x) \frac{t^{n}}{n !} \text {. }
$$

When $x=0, E_{n}=E_{n}(0)$ are called the Euler numbers. We observe that $E_{n}(x)=\sum_{j=0}^{n}\left(\begin{array}{c}n \\ j\end{array}\right) E_{n-j} x^{j},(\mathrm{~d} / \mathrm{d} x) E_{n}(x)=$ $n E_{n-1}(x), E_{n}(x+1)+E_{n}(x)=2 x^{n}$. The first few terms of $E_{n}$ are given by

$$
\begin{aligned}
E_{0} & =1, \\
E_{1} & =-\frac{1}{2}, \\
E_{3} & =\frac{1}{4} \\
E_{5} & =-\frac{1}{2} \\
E_{7} & =\frac{17}{8}, \\
E_{9} & =-\frac{31}{2}, \\
& \ldots, \\
E_{2 k} & =0, \quad(k \geq 1) .
\end{aligned}
$$

More generally, for any nonnegative integer $r$, the Euler polynomials $E_{n}^{(r)}(x)$ of order $r$ are given by

$$
\left(\frac{2}{e^{t}+1}\right)^{r} e^{x t}=\sum_{n=0}^{\infty} E_{n}^{(r)}(x) \frac{t^{n}}{n !}
$$

The Genocchi polynomials $G_{n}(x)$ are defined by

$$
\frac{2 t}{e^{t}+1} e^{x t}=\sum_{n=0}^{\infty} G_{n}(x) \frac{t^{n}}{n !}
$$

When $x=0, G_{n}=G_{n}(0)$ are called the Genocchi numbers. We observe that $G_{n}(x)=\sum_{j=0}^{n}\left(\begin{array}{c}n \\ j\end{array}\right)$ $G_{n-j} x^{j},(\mathrm{~d} / \mathrm{d} x) G_{n}(x)=n G_{n-1}(x), \quad G_{n}(x+1)+G_{n}(x)$ $=2 n x^{n-1}$, and $\operatorname{deg} G_{n}(x)=n-1$, for $n \geq 1$. Note that $G_{0}(x)=0$. The first few terms of $G_{n}$ are given by

$$
\begin{aligned}
G_{0} & =0, \\
G_{1} & =1, \\
G_{2} & =-1, \\
G_{4} & =1, \\
G_{6} & =-3, \\
G_{8} & =17, \\
G_{10} & =-155, \\
G_{12} & =2073, \\
& \cdots \\
G_{2 k+1} & =0, \quad(k \geq 1) .
\end{aligned}
$$

More generally, for any nonnegative integer $r$, the Genocchi polynomials $G_{n}^{(r)}(x)$ of order $r$ are given by

$$
\left(\frac{2 t}{e^{t}+1}\right)^{r} e^{x t}=\sum_{n=0}^{\infty} G_{n}^{(r)}(x) \frac{t^{n}}{n !}
$$

Observe here that $G_{0}^{(r)}(x)=G_{1}^{(r)}(x)=\cdots=G_{r-1}^{(r)}(x)=0$ and that $G_{n}^{(r)}(x)=(n ! /(n-r) !) E_{n-r}^{(r)}(x)$ has degree $n-r$, for $n \geq r$.

For any nonzero real number $\lambda$, the degenerate exponentials are given by $([3,5,6])$

$$
\begin{aligned}
& e_{\lambda}^{x}(t)=(1+\lambda t)^{x / \lambda}=\sum_{n=0}^{\infty}(x)_{n, \lambda} \frac{t^{n}}{n !} \\
& e_{\lambda}(t)=e_{\lambda}^{1}(t)=(1+\lambda t)^{1 / \lambda}=\sum_{n=0}^{\infty}(1)_{n, \lambda} \frac{t^{n}}{n !} .
\end{aligned}
$$

Carlitz [16] introduced a degenerate version of the Euler polynomials $E_{n}(x)$, called the degenerate Euler polynomials and denoted by $\mathscr{E}_{n, \lambda}(x)$, which are given by

$$
\frac{2}{e_{\lambda}(t)+1} e_{\lambda}^{x}(t)=\sum_{n=0}^{\infty} \mathscr{E}_{n, \lambda}(x) \frac{t^{n}}{n !}
$$

For $x=0, \mathscr{E}_{n, \lambda}=\mathscr{E}_{n, \lambda}(0)$ are called the degenerate Euler numbers. 
More generally, for any nonnegative integer $r$, the degenerate Euler polynomials $\mathscr{E}_{n, \lambda}^{(r)}(x)$ of order $r$ are given by

$$
\left(\frac{2}{e_{\lambda}(t)+1}\right)^{r} e_{\lambda}^{x}(t)=\sum_{n=0}^{\infty} \mathscr{E}_{n, \lambda}^{(r)}(x) \frac{t^{n}}{n !}
$$

A degenerate version of the Genocchi polynomials $G_{n}(x)$, called the degenerate Genocchi polynomials and denoted by $\mathscr{G}_{n, \lambda}(x)$, are given by ([17])

$$
\frac{2 t}{e_{\lambda}(t)+1} e_{\lambda}^{x}(t)=\sum_{n=0}^{\infty} \mathscr{G}_{n, \lambda}(x) \frac{t^{n}}{n !}
$$

For $x=0, \mathscr{G}_{n, \lambda}=\mathscr{G}_{n, \lambda}(0)$ are called the degenerate Genocchi numbers. Note that $\mathscr{G}_{0, \lambda}(x)=0$.

More generally, for any nonnegative integer $r$, the degenerate Genocchi polynomials $\mathscr{G}_{n, \lambda}^{(r)}(x)$ of order $r$ are given by $([17])$

$$
\left(\frac{2 t}{e_{\lambda}(t)+1}\right)^{r} e_{\lambda}^{x}(t)=\sum_{n=0}^{\infty} \mathscr{G}_{n, \lambda}^{(r)}(x) \frac{t^{n}}{n !}
$$

For $x=0, \mathscr{G}_{n, \lambda}^{(r)}=\mathscr{G}_{n, \lambda}^{(r)}(0)$ are called the degenerate Genocchi numbers of order $r$. Observe that $\mathscr{G}_{0, \lambda}^{(r)}(x)=\mathscr{G}_{1, \lambda}^{(r)}(x)=\cdots=\mathscr{G}_{r-1, \lambda}^{(r)}(x)=0$ and that $\mathscr{G}_{n, \lambda}^{(r)}(x)=$ $(n ! /(n-r) !) \mathscr{E}_{n-r, \lambda}^{(r)}(x)$ has degree $n-r$, for $n \geq r$.

We remark that $\mathscr{G}_{n, \lambda}(x) \longrightarrow G_{n}(x)$ and $\mathscr{G}_{n, \lambda}^{(r)}(x)$ $\longrightarrow G_{n}^{(r)}(x)$, as $\lambda$ tends to 0 .

We recall some notations and facts about forward differences. Let $f$ be any complex-valued function of the real variable $x$. Then, for any real number $a$, the forward difference $\Delta_{a}$ is given by

$$
\Delta_{a} f(x)=f(x+a)-f(x) .
$$

If $a=1$, then we let

$$
\Delta f(x)=\Delta_{1} f(x)=f(x+1)-f(x) .
$$

We also need

$$
\widetilde{\Delta} f(x)=f(x+1)+f(x) .
$$

It is necessary to note that

$$
\Delta_{a} \widetilde{\Delta}=\widetilde{\Delta} \Delta_{a} .
$$

In general, the $n^{\text {th }}$ order forward differences are given by

$$
\Delta_{a}^{n} f(x)=\sum_{i=0}^{n}\left(\begin{array}{l}
n \\
i
\end{array}\right)(-1)^{n-i} f(x+i a) .
$$

For $a=1$, we have

$$
\Delta^{n} f(x)=\sum_{i=0}^{n}\left(\begin{array}{l}
n \\
i
\end{array}\right)(-1)^{n-i} f(x+i) .
$$

It is easy to see that

$$
\widetilde{\Delta}^{n} f(x)=\sum_{i=0}^{n}\left(\begin{array}{l}
n \\
i
\end{array}\right) f(x+i) .
$$

Finally, we recall that the Stirling numbers of the second kind $S_{2}(n, k)$ are given by

$$
\frac{1}{k !}\left(e^{t}-1\right)^{k}=\sum_{n=k}^{\infty} S_{2}(n, k) \frac{t^{n}}{n !}, \quad k \geq 0 .
$$

\section{Review of Umbral Calculus}

Here, we will briefly go over very basic facts about umbral calculus. For more details on this, we recommend the reader to refer to [18-20]. Let $\mathbb{C}$ be the field of complex numbers. Then, $\mathscr{F}$ denotes the algebra of formal power series in $t$ over $\mathbb{C}$ given by

$$
\mathscr{F}=\left\{f(t)=\sum_{k=0}^{\infty} a_{k} \frac{t^{k}}{k !} \mid, \quad a_{k} \in \mathbb{C}\right\},
$$

and $\mathbb{P}=\mathbb{C}[x]$ indicates the algebra of polynomials in $x$ with coefficients in $\mathbb{C}$.

The set of all linear functionals on $\mathbb{P}$ is a vector space as usual and denoted by $\mathbb{P}^{*}$. Let $\langle L \mid p(x)\rangle$ denote the action of the linear functional $L$ on the polynomial $p(x)$.

For $f(t) \in \mathscr{F}$ with $f(t)=\sum_{k=0}^{\infty} a_{k}\left(t^{k} / k !\right)$, we define the linear functional on $\mathbb{P}$ by

$$
\left\langle f(t) \mid x^{k}\right\rangle=a_{k} .
$$

From (25), we note that

$$
\left\langle t^{k} \mid x^{n}\right\rangle=n ! \delta_{n, k}, \quad(n, k \geq 0),
$$

where $\delta_{n, k}$ is Kronecker's symbol.

Some remarkable linear functionals are as follows:

$$
\begin{aligned}
\left\langle e^{y t} \mid p(x)\right\rangle & =p(y), \\
\left\langle e^{y t}-1 \mid p(x)\right\rangle & =p(y)-p(0), \\
\left\langle\frac{e^{y t}-1}{t} \mid p(x)\right\rangle & =\int_{0}^{y} p(u) \mathrm{d} u .
\end{aligned}
$$

Let

$$
f_{L}(t)=\sum_{k=0}^{\infty}\left\langle L \mid x^{k}\right\rangle \frac{t^{k}}{k !}
$$

Then, by (25) and (28), we get

$$
\left\langle f_{L}(t) \mid x^{n}\right\rangle=\left\langle L \mid x^{n}\right\rangle .
$$

That is, $f_{L}(t)=L$, as linear functionals on $\mathbb{P}^{*}$, shows the map $L \mapsto f_{L}(t)$ from $\mathbb{P}^{*}$ onto $\mathscr{F}$ is one-to-one. Additionally, the map $L \mapsto f_{L}(t)$ is linear and also onto in view of (25) and (28). Thus, it is a vector space isomorphism from $\mathbb{P}^{*}$ onto $\mathscr{F}$.

Henceforth, $\mathscr{F}$ denotes both the algebra of formal power series in $t$ and the vector space of all linear functionals on $\mathbb{P}$. $\mathscr{F}$ is called the umbral algebra, and the umbral calculus is the study of umbral algebra. For each nonnegative integer $k$, the differential operator $t^{k}$ on $\mathbb{P}$ is defined by

$$
t^{k} x^{n}= \begin{cases}(n)_{k} x^{n-k}, & \text { if } k \leq n, \\ 0, & \text { if } k>n .\end{cases}
$$

Extending (30) linearly, any power series 


$$
f(t)=\sum_{k=0}^{\infty} \frac{a_{k}}{k !} t^{k} \in \mathscr{F},
$$

gives the differential operator on $\mathbb{P}$ defined by

$$
f(t) x^{n}=\sum_{k=0}^{n}\left(\begin{array}{l}
n \\
k
\end{array}\right) a_{k} x^{n-k}, \quad n \geq 0 .
$$

It should be observed that, for any formal power series $f(t)$ and any polynomial $p(x)$, we have

$$
\langle f(t) \mid p(x)\rangle=\langle 1 \mid f(t) p(x)\rangle=\left.f(t) p(x)\right|_{x=0} .
$$

Here, we note that an element $f(t)$ of $\mathscr{F}$ is a formal power series, a linear functional, and a differential operator. Some notable differential operators are as follows:

$$
\begin{aligned}
e^{y t} p(x) & =p(x+y), \\
\left(e^{y t}-1\right) p(x) & =p(x+y)-p(x), \\
\frac{e^{y t}-1}{t} p(x) & =\int_{x}^{x+y} p(u) \mathrm{d} u .
\end{aligned}
$$

The order $o(f(t))$ of the power series $f(t)(\neq 0)$ is the smallest integer for which $a_{k}$ does not vanish. If $o(f(t))=0$, then $f(t)$ is called an invertible series. If $o(f(t))=1$, then $f(t)$ is called a delta series.

For $f(t), g(t) \in \mathscr{F}$ with $o(f(t))=1$ and $o(g(t))=0$, there exists a unique sequence $s_{n}(x)\left(\operatorname{deg} s_{n}(x)=n\right)$ of polynomials, such that

$$
\left\langle g(t) f(t)^{k} \mid s_{n}(x)\right\rangle=n ! \delta_{n, k}, \quad(n, k \geq 0)
$$

The sequence $s_{n}(x)$ is said to be the Sheffer sequence for $(g(t), f(t))$, which is denoted by $s_{n}(x) \sim(g(t), f(t))$. We observe from (35) that

$$
s_{n}(x)=\frac{1}{g(t)} p_{n}(x),
$$

where $p_{n}(x)=g(t) s_{n}(x) \sim(1, f(t))$.

In particular, if $s_{n}(x) \sim(g(t), t)$, then $p_{n}(x)=x^{n}$, and hence,

$$
s_{n}(x)=\frac{1}{g(t)} x^{n}
$$

It is well known that $s_{n}(x) \sim(g(t), f(t))$ if and only if

$$
\frac{1}{g(\bar{f}(t))} e^{x \bar{f}(t)}=\sum_{k=0}^{\infty} \frac{s_{k}(x)}{k !} t^{k}
$$

for all $x \in \mathbb{C}$, where $\bar{f}(t)$ is the compositional inverse of $f(t)$, such that $\bar{f}(f(t))=f(\bar{f}(t))=t$.

The following equations (39)-(41) are equivalent to the fact that $s_{n}(x)$ is Sheffer for $(g(t), f(t))$, for some invertible $g(t)$ :

$$
\begin{aligned}
& f(t) s_{n}(x)=n s_{n-1}(x), \quad(n \geq 0), \\
& s_{n}(x+y)=\sum_{j=0}^{n}\left(\begin{array}{l}
n \\
j
\end{array}\right) s_{j}(x) p_{n-j}(y),
\end{aligned}
$$

with $p_{n}(x)=g(t) s_{n}(x)$,

$$
s_{n}(x)=\sum_{j=0}^{n} \frac{1}{j !}\left\langle g(\bar{f}(t))^{-1} \bar{f}(t)^{j} \mid x^{n}\right\rangle x^{j} .
$$
have

For $s_{n}(x) \sim(g(t), f(t))$ and $r_{n}(x) \sim(h(t), l(t))$, we

$$
s_{n}(x)=\sum_{k=0}^{n} C_{n, k} r_{k}(x), \quad n \geq 0,
$$

where

$$
C_{n, k}=\frac{1}{k !}\left\langle\frac{h(\bar{f}(t))}{g(\bar{f}(t))} l(\bar{f}(t))^{k} \mid x^{n}\right\rangle .
$$

\section{Representation by Degenerate Genocchi Polynomials}

Our interest here is to derive formulas expressing any polynomial in terms of the degenerate Genocchi polynomials.

From (11), we first observe that

$$
(x)_{n, \lambda} \sim\left(1, f(t)=\frac{1}{\lambda}\left(e^{\lambda t}-1\right)\right) .
$$

From (39), we note that

$$
f(t)(x)_{n, \lambda}=n(x)_{n-1, \lambda} .
$$

It is immediate to see from (14) that

$$
\widetilde{\Delta} \mathscr{G}_{n, \lambda}(x)=\mathscr{G}_{n, \lambda}(x+1)+\mathscr{G}_{n, \lambda}(x)=2 n(x)_{n-1, \lambda} \text {. }
$$

Now, we assume that $p(x) \in \mathbb{C}[x]$ has degree $n$, and write $\quad p(x)=\sum_{k=1}^{n+1} a_{k} \mathscr{G}_{n, \lambda}(x)$. Let $a(x)=p(x+1)+$ $p(x)=\widetilde{\Delta} p(x)$. Then, from (45) and (46), we have

$$
\begin{aligned}
a(x) & =\sum_{k=1}^{n+1} a_{k}\left(\mathscr{G}_{k, \lambda}(x+1)+\mathscr{G}_{k, \lambda}(x)\right) \\
& =2 \sum_{k=1}^{n+1} a_{k} k(x)_{k-1, \lambda} \\
& =2 f(t) \sum_{k=1}^{n+1} a_{k}(x)_{k, \lambda} .
\end{aligned}
$$

For $k \geq 1$, from (45) and (47), we obtain

$$
\begin{aligned}
(f(t))^{k-1} a(x) & =2(f(t))^{k} \sum_{l=1}^{n+1} a_{l}(x)_{l, \lambda} \\
& =2 \sum_{l=k}^{n+1} l(l-1) \cdots(l-k+1) a_{l}(x)_{l-k, \lambda} .
\end{aligned}
$$

Letting $x=0$ in (48), we finally get

$$
\begin{aligned}
a_{k} & =\left.\frac{1}{2 k !}(f(t))^{k-1} a(x)\right|_{x=0} \\
& =\frac{1}{2 k !}\left\langle(f(t))^{k-1} \mid a(x)\right\rangle, \quad(1 \leq k \leq n+1) .
\end{aligned}
$$


An alternative expression of (49) is given by

$$
\begin{aligned}
a_{k} & =\left.\frac{1}{2 k ! \lambda^{k-1}} \Delta_{\lambda}^{k-1} a(x)\right|_{x=0} \\
& =\frac{1}{2 k ! \lambda^{k-1}}\left(\Delta_{\lambda}^{k-1} p(1)+\Delta_{\lambda}^{k-1} p(0)\right) \\
& =\frac{1}{2 k ! \lambda^{k-1}} \Delta_{\lambda}^{k-1} \tilde{\Delta} p(0),
\end{aligned}
$$

since $f(t) a(x)=(1 / \lambda)\left(e^{\lambda t}-1\right) a(x)=(1 / \lambda) \Delta_{\lambda} a(x)$.

From (50), we have another alternative expression of (49) which is given by

$$
\begin{aligned}
a_{k} & =\left.\frac{1}{2 k ! \lambda^{k-1}} \Delta_{\lambda}^{k-1} a(x)\right|_{x=0} \\
& =\left.\frac{1}{2 k ! \lambda^{k-1}} \sum_{l=0}^{k-1}\left(\begin{array}{c}
k-1 \\
l
\end{array}\right)(-1)^{k-1-l} a(x+l \lambda)\right|_{x=0} \\
& =\frac{1}{2 k ! \lambda^{k-1}} \sum_{l=0}^{k-1}\left(\begin{array}{c}
k-1 \\
l
\end{array}\right)(-1)^{k-1-l}(p(1+l \lambda)+p(l \lambda)) .
\end{aligned}
$$

From (49), we get yet another expression of $a_{k}$ as follows:

$$
\begin{aligned}
a_{k} & =\left.\frac{1}{2 k \lambda^{k-1}} \frac{1}{(k-1) !}\left(e^{\lambda t}-1\right)^{k-1} a(x)\right|_{x=0} \\
& =\frac{1}{2 k} \sum_{l=k-1}^{n} S_{2}(l, k-1) \frac{\lambda^{l-k+1}}{l !}\left(p^{(l)}(1)+p^{(l)}(0)\right),
\end{aligned}
$$

where $p^{(l)}(x)=(\mathrm{d} / \mathrm{d} x)^{l} p(x)$.

Finally, from (49)-(52), we get the following theorem.where $f(t)=(1 / \lambda)\left(e^{\lambda t}-1\right)$.

Theorem 1. Let $p(x) \in \mathbb{C}[x], \operatorname{deg} p(x)=n$. Then, we have $p(x)=\sum_{k=1}^{n+1} a_{k} \mathscr{G}_{k, \lambda}(x)$, where $a_{k}$ 's are given by the following various expressions:

$$
\begin{aligned}
& a_{k}=\left.\frac{1}{2 k !}(f(t))^{k-1}(p(x+1)+p(x))\right|_{x=0} \\
&=\frac{1}{2 k ! \lambda^{k-1}}\left\langle\left(e^{\lambda t}-1\right)^{k-1} \mid p(x+1)+p(x)\right\rangle \\
&=\frac{1}{2 k ! \lambda^{k-1}} \Delta_{\lambda}^{k-1} \widetilde{\Delta} p(0) \\
&=\frac{1}{2 k ! \lambda^{k-1}} \sum_{l=0}^{k-1}\left(\begin{array}{c}
k-1 \\
l=1
\end{array}\right)(-1)^{k-1-l}(p(1+l \lambda)+p(l \lambda)) \\
&=\frac{1}{2 k} \sum_{l=k-1}^{n} S_{2}(l, k-1) \frac{\lambda^{l-k+1}}{l !}\left(p^{(l)}(1)+p^{(l)}(0)\right) \\
& \quad \text { for } k=1,2, \ldots, n+1,
\end{aligned}
$$

Remark 1. Let $p(x) \in \mathbb{C}[x]$, with $\operatorname{deg} p(x)=n$. Write $p(x)=\sum_{k=1}^{n+1} a_{k} G_{k}(x)$. As $\lambda$ tends to $0, f(t) \longrightarrow t$. Thus, we get the following result, namely, we have

$$
a_{k}=\frac{1}{2 k !}\left(p^{(k-1)}(1)+p^{(k-1)}(0)\right), \quad \text { for } k=1,2, \ldots, n+1 \text {. }
$$

Remark 2. The formulas in (1) and (54) and analogous formula for representation by Euler polynomials have been applied to many polynomials in order to obtain interesting identities for certain special polynomials and numbers. Some of the polynomials that have been considered are as follows:

(a)

$$
\begin{aligned}
& \sum B_{i_{1}}(x) \cdots B_{i_{r}}(x) E_{j_{1}}(x) \cdots E_{j_{s}}(x) \\
& G_{k_{1}+1}(x) \cdots G_{k_{t}+1}(x) x^{l}
\end{aligned}
$$

Where the sum is over all nonnegative integers $i_{1}, \ldots, i_{r}, j_{1}, \ldots, j_{s}, k_{1}, \ldots, k_{t}, l, \quad$ such that $i_{1}+\cdots+i_{r}+j_{1}+\cdots+j_{s}+k_{1}+\cdots+k_{t}+l=n$, and $r, s, t, l$ are the nonnegative integers with $r+s+t \geq 1$.

(b)

$$
\sum \frac{B_{i_{1}}(x) \cdots B_{i_{r}}(x) E_{j_{1}}(x) \cdots E_{j_{s}}(x) G_{k_{1}+1}(x) \cdots G_{k_{t}+1}(x) x^{l}}{i_{1} ! \cdots i_{r} ! j_{1} ! \cdots j_{s} !\left(k_{1}+1\right) ! \cdots\left(k_{t}+1\right) ! l !},
$$

Where the sum is over all nonnegative integers $i_{1}, \ldots, i_{r}, j_{1}, \ldots, j_{s}, k_{1}, \ldots, k_{t}, l$, such that $i_{1}+\cdots+i_{r}+j_{1}+\cdots+j_{s}+k_{1}+\cdots+k_{t}+l=n$, and $r, s, t, l$ are the nonnegative integers with $r+s+t \geq 1$.

(c)

$$
\sum \frac{B_{i_{1}}(x) \cdots B_{i_{r}}(x) E_{j_{1}}(x) \cdots E_{j_{s}}(x) G_{k_{1}+1}(x) \cdots G_{k_{t}+1}(x) x^{l}}{i_{1} \cdots i_{r} j_{1} \cdots j_{s}\left(k_{1}+1\right) \cdots\left(k_{t}+1\right) l},
$$

Where the sum is over all positive integers $i_{1}, \ldots, i_{r}, j_{1}, \ldots, j_{s}, l$ and nonnegative integers $k_{1}, \ldots, k_{t}$, such that $i_{1}+\cdots+i_{r}+j_{1}+\cdots+j_{s}+k_{1}+\cdots+k_{t}+l=n$, and $r, s, t, l$ are the nonnegative integers with $r+s+t \geq 1$.

\section{Representation by Higher-Order Degenerate Genocchi Polynomials}

Our interest here is to derive formulas expressing any polynomial in terms of the higher-order degenerate Genocchi polynomials.

As we noted in (45), we have $f(t)(x)_{n, \lambda}=n(x)_{n-1, \lambda}$, with $f(t)=(1 / \lambda)\left(e^{\lambda t}-1\right)$. Let $g(t)=e^{t}+1$.

It is immediate to see from (15) that

$$
\widetilde{\Delta} \mathscr{G}_{n, \lambda}^{(r)}(x)=\mathscr{G}_{n, \lambda}^{(r)}(x+1)+\mathscr{G}_{n, \lambda}^{(r)}(x)=2 n \mathscr{G}_{n-1, \lambda}^{(r-1)}(x)
$$

Repeated application of (58) gives us 


$$
\begin{aligned}
\widetilde{\Delta}^{r} \mathscr{G}_{n, \lambda}^{(r)}(x) & =(2 n)(2(n-1)) \cdots(2(n-r+1)) \mathscr{G}_{n-r, \lambda}^{(0)}(x) \\
& =2^{r} n(n-1) \cdots(n-r+1)(x)_{n-r, \lambda}, \quad(n \geq r) .
\end{aligned}
$$

Now, we assume that $p(x) \in \mathbb{C}[x]$ has degree $n$, and write $p(x)=\sum_{k=r}^{n+r} a_{k} \mathscr{G}_{k, \lambda}^{(r)}(x)$. Then, from (59), we observe that

$$
\begin{aligned}
g(t)^{r} p(x) & =\widetilde{\Delta}^{r} p(x)=\sum_{l=r}^{n+r} a_{l} \widetilde{\Delta}^{r} \mathscr{G}_{l, \lambda}^{(r)}(x) \\
& =2^{r} \sum_{l=r}^{n+r} l(l-1) \cdots(l-r+1) a_{l}(x)_{l-r, \lambda} .
\end{aligned}
$$

By using (45) and (60), for $k \geq 0$, we observe that

$$
\begin{aligned}
f(t)^{k} g(t)^{r} p(x)= & 2^{r} \sum_{l=r+k}^{n+r} l(l-1) \cdots \\
& (l-r-k+1) a_{l}(x)_{l-r-k, \lambda} .
\end{aligned}
$$

By evaluating (61) at $x=0$, we obtain

$$
\begin{aligned}
a_{k+r} & =\left.\frac{1}{2^{r}(k+r) !} f(t)^{k} g(t)^{r} p(x)\right|_{x=0} \\
& =\frac{1}{2^{r}(k+r) !}\left\langle f(t)^{k} \mid g(t)^{r} p(x)\right\rangle, \quad(0 \leq k \leq n) .
\end{aligned}
$$

Several alternative expressions of (62) follow from (20) and (22), which are given by

$$
\begin{aligned}
a_{k} & =\left.\frac{1}{2^{r} k !} g(t)^{r} f(t)^{k-r} p(x)\right|_{x=0} \\
& =\frac{1}{2^{r} k ! \lambda^{k-r}} \widetilde{\Delta}^{r} \Delta_{\lambda}^{k-r} p(0) \\
& =\frac{1}{2^{r} k ! \lambda^{k-r}} \sum_{j=0}^{r} \sum_{l=0}^{k-r}\left(\begin{array}{c}
r \\
j
\end{array}\right)\left(\begin{array}{c}
k-r \\
l
\end{array}\right)(-1)^{k-r-l} p(j+l \lambda) \\
& =\left.\frac{1}{2^{r} k ! \lambda^{k-r}} \tilde{\Delta}^{r-1} \Delta_{\lambda}^{k-r}(p(x+1)+p(x))\right|_{x=0}, \quad r \leq k \leq n+r .
\end{aligned}
$$

Another expression for $a_{k}$ follows from (23):

$$
\begin{aligned}
a_{k}= & \left.\frac{1}{2^{r} \lambda^{k-r} k !} \sum_{j=0}^{r}\left(\begin{array}{c}
r \\
j
\end{array}\right) \Delta_{\lambda}^{k-r} p(x+j)\right|_{x=0} \\
= & \left.\frac{(k-r) !}{2^{r} \lambda^{k-r} k !} \sum_{j=0}^{r}\left(\begin{array}{c}
r \\
j
\end{array}\right) \frac{1}{(k-r) !}\left(e^{\lambda t}-1\right)^{k-r} p(x+j)\right|_{x=0} \\
= & \frac{(k-r) !}{2^{r} k !} \sum_{j=0}^{r} \sum_{l=k-r}^{n}\left(\begin{array}{c}
r \\
j
\end{array}\right) \frac{\lambda^{l-k+r}}{l !} S_{2} \\
& \cdot(l, k-r) p^{(l)}(j), \quad(r \leq k \leq n+r) .
\end{aligned}
$$

Summarizing the results so far, from (62) and (64), we obtain the following theorem.

$$
p(x)=\sum_{k=r}^{n+r} a_{k} \mathscr{G}_{k, \lambda}^{(r)}(x),
$$

where $a_{k}$ 's are given by the following various expressions:

Theorem 2. Let $p(x) \in \mathbb{C}[x], \operatorname{deg} p(x)=n$. Let $g(t)=$ $e^{t}+1, f(t)=(1 / \lambda)\left(e^{\lambda t}-1\right)$. Then, we have

$$
\begin{aligned}
a_{k}= & \left.\frac{1}{2^{r} k !} g(t)^{r} f(t)^{k-r} p(x)\right|_{x=0} \\
= & \frac{1}{2^{r} k ! \lambda^{k-r}} \widetilde{\Delta}^{r} \Delta_{\lambda}^{k-r} p(0) \\
= & \frac{1}{2^{r} k ! \lambda^{k-r}} \sum_{j=0}^{r} \sum_{l=0}^{k-r}\left(\begin{array}{c}
r \\
j
\end{array}\right)\left(\begin{array}{c}
k-r \\
l
\end{array}\right)(-1)^{k-r-l} p(j+l \lambda) \\
= & \left.\frac{1}{2^{r} k ! \lambda^{k-r}} \sum_{j=0}^{r-1}\left(\begin{array}{c}
r-1 \\
j
\end{array}\right) \Delta_{\lambda}^{k-r}(p(x+j+1)+p(x+j))\right|_{x=0} \\
= & \frac{(k-r) !}{2^{r} k !} \sum_{j=0}^{r} \sum_{l=k-r}^{n}\left(\begin{array}{c}
r \\
j
\end{array}\right) \frac{\lambda^{l-k+r}}{l !} S_{2} \\
& (l, k-r) p^{(l)}(j), \quad(r \leq k \leq n+r) .
\end{aligned}
$$

Remark 3. Let $p(x) \in \mathbb{C}[x]$, with $\operatorname{deg} p(x)=n$. Write $p(x)=\sum_{k=r}^{n+r} a_{k} G_{k}^{(r)}(x)$. As $\lambda$ tends to $0, f(t) \longrightarrow t$. Thus, from Theorem 2 , we have the following result.

$$
\begin{aligned}
a_{k} & =\frac{1}{2^{r} k !} g(t)^{r} p^{(k-r)}(0) \\
& =\frac{1}{2^{r} k !} \sum_{j=0}^{r-1}\left(\begin{array}{c}
r-1 \\
j
\end{array}\right)\left(p^{(k-r)}(j+1)+p^{(k-r)}(j)\right) \\
& =\frac{1}{2^{r} k !} \sum_{j=0}^{r}\left(\begin{array}{c}
r \\
j
\end{array}\right) p^{(k-r)}(j), \quad(r \leq k \leq n+r) .
\end{aligned}
$$

\section{Examples}

(a) Here, we illustrate Theorem 1, with $p(x)=G_{n}(x)$. Let $G_{n}(x)=\sum_{k=1}^{n+1} a_{k} \mathscr{G}_{k, \lambda}(x)$.

For $k$ with $1 \leq k \leq n+1$, we have

$$
\begin{aligned}
a_{k} & =\frac{1}{2 k ! \lambda^{k-1}}\left\langle\left(e^{\lambda t}-1\right)^{k-1} \mid G_{n}(x+1)+G_{n}(x)\right\rangle \\
& =\frac{1}{2 k ! \lambda^{k-1}}\left\langle\left(e^{\lambda t}-1\right)^{k-1} \mid 2 n x^{n-1}\right\rangle \\
& =\left.\frac{n}{k ! \lambda^{k-1}} \Delta_{\lambda}^{k-1} x^{n-1}\right|_{x=0} \\
& =\frac{n}{k ! \lambda^{k-1}} \Delta_{\lambda}^{k-1} 0^{n-1} .
\end{aligned}
$$




$$
G_{n}(x)=\sum_{k=1}^{n+1} \frac{n}{k ! \lambda^{k-1}} \Delta_{\lambda}^{k-1} 0^{n-1} \mathscr{G}_{k, \lambda}(x) .
$$

(b) Here, we illustrate Theorem 1, for $p(x)=\sum_{k=1}^{n-1}(1 / k(n-k)) B_{k}(x) B_{n-k}(x),(n \geq 2)$. For this, we first recall from [8] that

$$
\begin{aligned}
p(x)= & \sum_{k=0}^{n-2}\left(\frac{2\left(\begin{array}{c}
n \\
k
\end{array}\right)}{n}\left(H_{n-1}-H_{n-k-1}\right) B_{n-k}+\left(\begin{array}{c}
n-1 \\
k
\end{array}\right) \sum_{l=k+1}^{n-1} \frac{B_{l-k} B_{n-l}}{(l-k)(n-l)}+\frac{\left(\begin{array}{c}
n-1 \\
k
\end{array}\right) B_{n-1-k}}{n-1-k}\right) E_{k}(x) \\
& +\frac{n-1}{2} E_{n-2}(x)+\frac{2}{n} H_{n-1} E_{n}(x),
\end{aligned}
$$

Where $H_{n}=1+(1 / 2)+\cdots+(1 / n)$ is the harmonic number. Let $p(x)=\sum_{k=1}^{n+1} a_{k} \mathscr{G}_{k, \lambda}(x)$. For $k$, with $1 \leq k \leq n+1$, we obtain

$$
\begin{aligned}
2 k ! \lambda^{k-1} a_{k}= & \left\langle\left(e^{\lambda t}-1\right)^{k-1} \mid p(x+1)+p(x)\right\rangle \\
= & \sum_{m=0}^{n-2}\left(\frac{2\left(\begin{array}{c}
n \\
m
\end{array}\right)}{n}\left(H_{n-1}-H_{n-m-1}\right) B_{n-m}+\left(\begin{array}{c}
n-1 \\
m
\end{array}\right) \sum_{l=m+1}^{n-1} \frac{B_{l-m} B_{n-l}}{(l-m)(n-l)}+\frac{\left(\begin{array}{c}
n-1 \\
m
\end{array}\right) B_{n-1-m}}{n-1-m}\right) \\
& \left\langle\left(e^{\lambda t}-1\right)^{k-1} \mid E_{m}(x+1)+E_{m}(x)\right\rangle \\
& +\frac{n-1}{2}\left\langle\left(e^{\lambda t}-1\right)^{k-1} \mid E_{n-2}(x+1)+E_{n-2}(x)\right\rangle \\
& +\frac{2}{n} H_{n-1}\left\langle\left(e^{\lambda t}-1\right)^{k-1} \mid E_{n}(x+1)+E_{n}(x)\right\rangle .
\end{aligned}
$$

By proceeding as we did in (a), from (71), we see that

$$
\begin{aligned}
k ! \lambda^{k-1} a_{k}= & \sum_{m=0}^{n-2}\left(\begin{array}{c}
2\left(\begin{array}{c}
n \\
m
\end{array}\right) \\
n
\end{array}\left(H_{n-1}-H_{n-m-1}\right) B_{n-m}+\left(\begin{array}{c}
n-1 \\
m
\end{array}\right) \sum_{l=m+1}^{n-1} \frac{B_{l-m} B_{n-l}}{(l-m)(n-l)}\right. \\
& \left.+\frac{\left(\begin{array}{c}
n-1 \\
m
\end{array}\right) B_{n-1-m}}{n-1-m}\right) \Delta_{\lambda}^{k-1} 0^{m}+\frac{n-1}{2} \Delta_{\lambda}^{k-1} 0^{n-2}+\frac{2}{n} H_{n-1} \Delta_{\lambda}^{k-1} 0^{n} .
\end{aligned}
$$


Thus, from (72) and for $n \geq 2$, we have

$$
\begin{aligned}
& \sum_{k=1}^{n-1} \frac{1}{k(n-k)} B_{k}(x) B_{n-k}(x) \\
& =\sum_{k=1}^{n+1} \frac{1}{k ! \lambda^{k-1}}\left\{\sum_{m=0}^{n-2}\left(\frac{2\left(\begin{array}{c}
n \\
m
\end{array}\right)}{n}\left(H_{n-1}-H_{n-m-1}\right) B_{n-m}+\left(\begin{array}{c}
n-1 \\
m
\end{array}\right) \sum_{l=m+1}^{n-1} \frac{B_{l-m} B_{n-l}}{(l-m)(n-l)}+\frac{\left(\begin{array}{c}
n-1 \\
m
\end{array}\right) B_{n-1-m}}{n-1-m}\right)\right. \\
& \left.\cdot \Delta_{\lambda}^{k-1} 0^{m}+\frac{n-1}{2} \Delta_{\lambda}^{k-1} 0^{n-2}+\frac{2}{n} H_{n-1} \Delta_{\lambda}^{k-1} 0^{n}\right\} \mathscr{G}_{k, \lambda}(x) .
\end{aligned}
$$

(c) In [4], it is shown that the following identity holds for $n \geq 2$ :

$$
\begin{aligned}
& \sum_{k=1}^{n-1} \frac{1}{k(n-k)} E_{k}(x) E_{n-k}(x) \\
& =\sum_{k=0}^{n-2} \sum_{l=k+1}^{n-1} \frac{\left(\begin{array}{c}
n-1 \\
k
\end{array}\right)}{(l-k)(n-l)} E_{l-k} E_{n-l} E_{k}(x)+\frac{2}{n} H_{n-1} E_{n}(x),
\end{aligned}
$$

Where $H_{n}=1+(1 / 2)+\cdots+(1 / n)$ is the harmonic number.

Write

$\sum_{k=1}^{n-1} 1 / k(n-k) E_{k}(x) E_{n-k}(x)=\sum_{k=1}^{n+1} a_{k} \mathscr{G}_{k, \lambda}(x)$.

By proceeding similarly to (b), we obtain the following identity:

$$
\begin{aligned}
& \sum_{k=1}^{n-1} \frac{1}{k(n-k)} E_{k}(x) E_{n-k}(x) \\
& =\sum_{k=1}^{n+1} \frac{1}{k ! \lambda^{k-1}}\left(\sum_{m=0}^{n-2} \sum_{l=m+1}^{n-1} \frac{\left(\begin{array}{c}
n-1 \\
m
\end{array}\right)}{(l-m)(n-l)} E_{l-m} E_{n-l} \Delta_{\lambda}^{k-1} 0^{m}+\frac{2}{n} H_{n-1} \Delta_{\lambda}^{k-1} 0^{n}\right) \mathscr{G}_{k, \lambda}(x)
\end{aligned}
$$

(d) In [4], it is proved that the following identity is valid for $n \geq 2$ :

$$
\begin{aligned}
& \sum_{k=1}^{n-1} \frac{1}{k(n-k)} B_{k}(x) E_{n-k}(x) \\
& =\sum_{k=0}^{n-2}\left(\frac{1}{n}\left(\begin{array}{l}
n \\
k
\end{array}\right)\left(H_{n-1}-H_{n-k-1}\right) B_{n-k}-\frac{1}{2}\left(\begin{array}{c}
n-1 \\
k
\end{array}\right) \frac{E_{n-k-1}}{n-k-1}\right) E_{k}(x)+\frac{2}{n} H_{n-1} E_{n}(x) .
\end{aligned}
$$


Again, by proceeding analogously to (b), we get the following identity:

$$
\begin{aligned}
& \sum_{k=1}^{n-1} \frac{1}{k(n-k)} B_{k}(x) E_{n-k}(x) \\
& =\sum_{k=1}^{n+1} \frac{1}{k ! \lambda^{k-1}}\left\{\sum_{m=0}^{n-2}\left(\frac{1}{n}\left(\begin{array}{c}
n \\
m
\end{array}\right)\left(H_{n-1}-H_{n-m-1}\right) B_{n-m}-\frac{1}{2}\left(\begin{array}{c}
n-1 \\
m
\end{array}\right) \frac{E_{n-m-1}}{n-m-1}\right) \Delta_{\lambda}^{k-1} 0^{m}+\frac{2}{n} H_{n-1} \Delta_{\lambda}^{k-1} 0^{n}\right\} \mathscr{G}_{k, \lambda}(x) .
\end{aligned}
$$

(e) Nielsen [21, 22] expressed the product of a Bernoulli polynomial and an Euler polynomial in terms of

Euler polynomials, namely, for nonnegative integers $m$ and $n$,

$$
\begin{aligned}
& B_{m}(x) E_{n}(x) \\
& =m E_{m+n-1}(x)+\sum_{r=0}^{m}\left(\begin{array}{c}
m \\
r
\end{array}\right) B_{r} E_{m+n-r}(x)-\frac{m}{2} \sum_{s=0}^{n}\left(\begin{array}{l}
n \\
s
\end{array}\right) E_{s} E_{m+n-s-1}(x) .
\end{aligned}
$$

Again, in a similar way to (b), we can show that

$$
\begin{aligned}
& B_{m}(x) E_{n}(x) \\
& =\sum_{k=1}^{m+n+1} \frac{1}{k ! \lambda^{k-1}}\left\{m \Delta_{\lambda}^{k-1} 0^{m+n-1}+\sum_{r=0}^{m}\left(\begin{array}{c}
m \\
r
\end{array}\right) B_{r} \Delta_{\lambda}^{k-1} 0^{m+n-r}-\frac{m}{2} \sum_{s=0}^{n}\left(\begin{array}{c}
n \\
s
\end{array}\right) E_{s} \Delta_{\lambda}^{k-1} 0^{m+n-s-1}\right\} \mathscr{G}_{k, \lambda}(x) .
\end{aligned}
$$

We also represent $B_{m}(x) E_{n}(x)$ in terms of the degenerate Genocchi polynomials of order $r$. Indeed, from Theorem 2, we obtain three expressions:

$$
\begin{aligned}
B_{m}(x) E_{n}(x)= & \sum_{k=r}^{m+n+r} \frac{1}{2^{r} k ! \lambda^{k-r}}\left(\sum_{j=0}^{r} \sum_{l=0}^{k-r}\left(\begin{array}{c}
r \\
j
\end{array}\right)\left(\begin{array}{c}
k-r \\
l
\end{array}\right)(-1)^{k-r-l} \times B_{m}(j+l \lambda) E_{n}(j+l \lambda)\right) \mathscr{G}_{k, \lambda}^{(r)}(x) \\
= & \sum_{k=r}^{m+n+r} \frac{1}{2^{r-1} k ! \lambda^{k-r}} \sum_{j=0}^{r-1}\left(\begin{array}{c}
r-1 \\
j
\end{array}\right)\left\{m \Delta_{\lambda}^{k-r} j^{m+n-1}+\sum_{r=0}^{m}\left(\begin{array}{c}
m \\
r
\end{array}\right) B_{r} \Delta_{\lambda}^{k-r} j^{m+n-r}-\frac{m}{2} \sum_{s=0}^{n}\left(\begin{array}{l}
n \\
s
\end{array}\right) E_{s} \Delta_{\lambda}^{k-r} j^{m+n-s-1}\right\} \mathscr{G}_{k, \lambda}^{(r)}(x) \\
= & \sum_{k=r}^{m+n+r}\left\{\frac{(k-r) !}{2^{r} k !} \sum_{j=0}^{r} \sum_{l=k-r}^{m+n} \sum\left(\begin{array}{c}
r \\
j
\end{array}\right)\left(\begin{array}{c}
m \\
a
\end{array}\right)\left(\begin{array}{l}
n \\
b
\end{array}\right) \lambda^{l-k+r} \times S_{2}(l, k-r) B_{m-a}(j) E_{n-b}(j)\right\} \\
& \cdot \mathscr{G}_{k, \lambda}^{(r)}(x), \quad(r \leq k \leq n+r),
\end{aligned}
$$


Where the innermost sum in (80) is over all integers $a$ and $b$ satisfying $a+b=l, 0 \leq a \leq m$, and $0 \leq b \leq n$. (f) In (4.20) of [1], it is shown that the following identity holds for $n \geq s$ :

$$
\sum_{\substack{i_{1}+\cdots+i_{s}=n \\
i_{1}, \ldots, i_{s} \geq 1}} G_{i_{1}}(x) \ldots G_{i_{s}}(x)=\frac{1}{n+s} \sum_{l=1}^{s}\left(\begin{array}{c}
s \\
l
\end{array}\right)(-2)^{l-1} \sum_{\substack{j_{0}+j_{1}+\cdots+j_{s-l}=n+1-l \\
j_{0}, j_{1}, \ldots, j_{s-l} \geq 1}}\left(\begin{array}{c}
n+s \\
j_{0}
\end{array}\right) G_{j_{1}} \cdots G_{j_{s}} G_{j_{0}}(x) .
$$

Then, proceeding similarly to (b), we can show that

$$
\begin{aligned}
& \sum_{\substack{i_{1}+\cdots+i_{s}=n \\
i_{1}, \ldots, i_{s} \geq 1}} G_{i_{1}}(x) \ldots G_{i_{s}}(x) \\
& =\sum_{k=1}^{n-s+1}\left\{\frac{1}{k ! \lambda^{k-1}} \frac{1}{n+s} \sum_{l=1}^{s}\left(\begin{array}{c}
s \\
l
\end{array}\right)(-2)^{l-1} \times \sum_{\substack{j_{0}+j_{1}+\cdots+j_{s-l}=n+1-l \\
j_{0}, j_{1}, \ldots, j_{s-l} \geq 1}} j_{0}\left(\begin{array}{c}
n+s \\
j_{0}
\end{array}\right) G_{j_{1}} \cdots G_{j_{s}} \Delta_{\lambda}^{k-1} 0^{j_{0}-1}\right\} \mathscr{G}_{k, \lambda}(x) .
\end{aligned}
$$

In general, in terms of the degenerate Genocchi

polynomials of order $r$, we obtain the following:

$$
\sum_{\substack{i_{1}+\ldots+i_{s}=n \\
i_{1}, \ldots, i_{s} \geq 1}} G_{i_{1}}(x) \ldots G_{i_{s}}(x)=\sum_{k=r}^{n-s+r}\left\{\frac{1}{2^{r-1} k ! \lambda^{k-r}} \sum_{m=0}^{r-1}\left(\begin{array}{c}
r-1 \\
m
\end{array}\right) \frac{1}{n+s} \sum_{l=1}^{s}\left(\begin{array}{c}
s \\
l
\end{array}\right)(-2)^{l-1} \times \sum_{\substack{j_{0}+j_{1}+\cdots+j_{s-l}=n+1-l \\
j_{0}, j_{1}, \ldots, j_{s-l} \geq 1}} j_{0}\left(\begin{array}{c}
n+s \\
j_{0}
\end{array}\right) G_{j_{1}} \cdots G_{j_{s}} \Delta_{\lambda}^{k-r} m^{j_{0}-1}\right\} \mathscr{G}_{k, \lambda}^{(r)}(x) .
$$

\section{Conclusion and Future Work}

In this study, we were interested in representing any polynomial in terms of the degenerate Genocchi polynomials and of the higher-order degenerate Genocchi polynomials. We were able to derive formulas for such representations with the help of umbral calculus. By letting $\lambda$ tend to zero, we obtained the formulas for representations by the Genocchi polynomials and by the higher-order Genocchi polynomials. Furthermore, we illustrated the formulas with some examples.

Even though the method adopted in this study is simple, they are very useful and powerful. Indeed, as mentioned in Section 1, both a variant of Miki's identity and Faber-Pandharipande-Zagier (FPZ) identity follow from the identity in (2) that can be derived from the formulas in (1), involving only derivatives and integrals of the given polynomial, while all the other known proofs are quite involved.

It is one of our future research projects to continue to find formulas for representing polynomials in terms of some specific special polynomials and to apply those to discovering some interesting identities.

\section{Data Availability}

No data were used to support this study.

\section{Conflicts of Interest}

The authors declare that they have no conflicts of interest.

\section{Acknowledgments}

The authors like to thank Jangjeon Institute for Mathematical Sciences for the support of this research.

\section{References}

[1] Y. He, "Some new results on products of the Apostol-Genocchi polynomials," Journal of Computational Analysis and Applications, vol. 22, no. 4, pp. 591-600, 2017.

[2] L.-C. Jang, B. M. Kim, S.-K. Choi, C. S. Ryoo, and D. V. Dolgy, "Some explicit identities for the modified higher-order degenerate q-Euler polynomials and their zeroes," The Journal of Nonlinear Science and Applications, vol. 10, no. 5, pp. 25242538, 2017.

[3] D. S. Kim and T. Kim, "A note on a new type of degenerate Bernoulli numbers," Russian Journal of Mathematical Physics, vol. 27, no. 2, pp. 227-235, 2020.

[4] D. S. Kim, T. Kim, and T. Mansour, "Euler basis and the product of several Bernoulli and Euler polynomials," Advanced Studies in Contemporary Mathematics, vol. 24, no. 4, pp. 535-547, 2014. 
[5] H. K. Kim and W. A. Khan, "Some identities of a new type of degenerate poly-Frobenius-Euler polynomials and numbers," Proceedings of the Jangjeon Mathematical Society, vol. 24, pp. 33-45, 2021.

[6] T. Kim and D. S. Kim, "Some identities on truncated polynomials associated with degenerate Bell polynomials," Russian Journal of Mathematical Physics, vol. 28, no. 3, pp. 342-355, 2021.

[7] V. Kurt, "Some relation between the Bernstein polynomials and second kind Bernoulli polynomials," Advanced Studies in Contemporary Mathematics, vol. 23, no. 1, pp. 43-48, 2013.

[8] V. Kurt, "On the alternating sums and application to ApostolBernoulli polynomials," Proceedings of the Jangjeon Mathematical Society, vol. 16, no. 2, pp. 251-258, 2013.

[9] J. Pan and F. Yang, "Some convolution identities for Frobenius-Euler polynomials," Advances in Differential Equations, vol. 2017, no. 6, p. 16, 2017.

[10] D. S. Lee and H. K. Kim, "On the new type of degenerate polyGenocchi numbers and polynomials," Advances in Difference Equations, vol. 431, p. 15, 2020.

[11] I. M. Gessel, "On Miki's identity for Bernoulli numbers," Journal of Number Theory, vol. 110, no. 1, pp. 75-82, 2005.

[12] H. Miki, "A relation between Bernoulli numbers," Journal of Number Theory, vol. 10, no. 3, pp. 297-302, 1978.

[13] K. Shiratani and S. Yokoyama, "An application of p-adic convolutions," Memoirs of the Faculty of Science, Kyushu University. Series A, Mathematics, vol. 36, no. 1, pp. 73-83, 1982.

[14] G. V. Dunne and C. Schubert, "Bernoulli number identities from quantum field theory and topological string theory," Communications in Number Theory and Physics, vol. 7, no. 2, pp. 225-249, 2013.

[15] C. Faber and R. Pandharipande, "Hodge integrals and Gromov-Witten theory," Inventiones Mathematicae, vol. 139, no. 1, pp. 173-199, 2000.

[16] L. Carlitz, "Degenerate stirling, Bernoulli and eulerian numbers," Utilitas Mathematica, vol. 15, pp. 51-88, 1979.

[17] D. Lim, "Some identities of degenerate Genocchi polynomials," Bulletin of the Korean Mathematical Society, vol. 53, no. 2, pp. 569-579, 2016.

[18] R. Dere and Y. Simsek, "Applications of umbral algebra to some special polynomials," Advanced Studies in Contemporary Mathematics, vol. 22, no. 3, pp. 433-438, 2012.

[19] S. Roman, "The umbral calculus," Pure and Applied Mathematics, Vol. 111, Academic Press, Inc. [Harcourt Brace Jovanovich, Publishers], , New York, NY, USA, 1984.

[20] Y. Simsek, "Special numbers and polynomials including their generating functions in umbral analysis methods," Axioms, vol. 7, no. 2, p. 12, 2018.

[21] L. Carlitz and M. Sholander, "The product of two Eulerian polynomials," Mathematics Magazine, vol. 36, no. 1, pp. 37-41, 1963.

[22] N. Nielsen, Traité Élémentaire des Nombres de Bernoulli, Gauthier-Villars, Paris, France, 1923. 